\title{
Left ventricular assist device as destination therapy: Application of the payment-by-results approach for the device reimbursement
}

\author{
Andrea Messori, PharmD, ${ }^{\mathrm{a}}$ Sabrina Trippoli, PharmD, ${ }^{\mathrm{a}}$ Massimo Bonacchi, MD, ${ }^{\mathrm{b}}$ and Guido Sani, $\mathrm{MD}^{\mathrm{b}}$
}

\begin{abstract}
Objective: Value-based methods are increasingly used to reimburse therapeutic innovation, and the payment-byresults approach has been proposed for handling interventions with limited therapeutic evidence. Because most left ventricular assist devices are supported by preliminary efficacy data, we examined the effectiveness data of the HeartMate (Thoratec Corp, Pleasanton, CA) device to explore the application of the payment-byresults approach to these devices and to develop a model for handling reimbursements.
\end{abstract}

Methods: According to our model, after establishing the societal economic countervalue for each month of life saved, each patient treated with one such device is associated to the payment of this countervalue for every month of survival lived beyond the final date of estimated life expectancy without left ventricular assist devices. Our base-case analysis, which used the published data of 68 patients who received the HeartMate device, was run with a monthly countervalue of $€ 5000$, no adjustment for quality of life, and a baseline life expectancy of 150 days without left ventricular assist devices. Sensitivity analysis was aimed at testing the effect of quality of life adjustments and changes in life expectancy without device.

Results: In our base-case analysis, the mean total reimbursement per patient was $€ 82,426$ (range, $€ 0$ to $€ 250,000 ; \mathrm{N}=68$ ) generated as the sum of monthly payments. This average value was close to the current price of the HeartMate device $(€ 75,000)$. Sensitivity testing showed that the base-case reimbursement of $€ 82,426$ was little influenced by variations in life expectancy, whereas variations in utility had a more pronounced impact.

Conclusion: Our report delineates an innovative procedure for appropriately allocating economic resources in this area of invasive cardiology.

In handling therapeutic innovation, value-based methods ${ }^{1-3}$ are increasingly used to determine reimbursements that are proportional to the magnitude of the (incremental) clinical benefit of the innovative treatment. In Europe, threshold values of cost-effectiveness recognize up to $€ 5000$ (monthly countervalue [MCV]) to each month of survival gained or to each quality-adjusted month gained. Likewise, yearly thresholds are generally set at approximately $€ 50,000$ to $€ 60,000, \$ 50,000$ to $\$ 100,000$, or $£ 25,000$ to $£ 30,000 .^{2,3}$

Converting the clinical benefit into an economic countervalue requires that the typical (incremental) benefit resulting from the innovative treatment is supported by a sufficient evidence with a reasonably small statistical variability. On the other hand, many innovative treatments are characterized by premature therapeutic evidence on their effectiveness, sim-

From the Laboratory of Pharmacoeconomics, University Hospital of Careggi, ${ }^{a}$ Firenze; and Cardiac Surgery, Department of Medical-Surgical Critical Area, ${ }^{\mathrm{b}}$ University of Florence, Firenze, Italy.

Disclosures: M.B. and G.S. have on a single occasion received reimbursement of travel expenses from companies manufacturing LVADs.

Received for publication Oct 28, 2008; revisions received Jan 10, 2009; accepted for publication Feb 12, 2009; available ahead of print May 1, 2009.

Address for reprints: Andrea Messori, PharmD, Azienda Careggi, Viale Pieraccini 17, 50134 Firenze, Italy (E-mail: andreamessori@interfree.it).

J Thorac Cardiovasc Surg 2009;138:480-5

$0022-5223 / \$ 36.00$

Copyright (c) 2009 by The American Association for Thoracic Surgery doi:10.1016/j.jtcvs.2009.02.016 ply because the clinical research on that type of therapeutic innovation is still at an initial stage.

Determining reimbursements for innovative treatments with premature therapeutic evidence is a challenging task because regulatory agencies tend to be cautious in accepting the claimed degree of incremental benefit, whereas manufacturers tend to be more optimistic in predicting the outcome expected from their innovation. One solution to this controversy is the payment-by-results approach, ${ }^{4-6}$ wherein reimbursements are not estimated from the magnitude of the "average" benefit (as demonstrated in previous clinical trials) but are determined from the outcomes prospectively observed in individual patients (so that nonresponders generate no payments and responders generate payments).

Most left ventricular assist devices (LVADs) are currently supported by scarce data documenting the extent of their incremental benefit. ${ }^{7}$ Also the analyses on the cost-effectiveness of these devices tend to give conflicting indications. ${ }^{7}$ Thus, LVADs are good candidates for the application of the payment-by-results approach.

Briefly, the payment-by-results approach applied to LVADs is simply implemented through the monthly payment of an amount of money equal to MCV for every month of survival lived by the patient beyond the final date of his or her life expectancy without an LVAD (predicted at baseline, ie, before implantation).

In this article, we have used the clinical information already published on the effectiveness of the HeartMate 


\section{Abbreviations and Acronyms \\ AST = additional survival time \\ bLE = baseline life expectancy \\ indST $=$ individual survival time \\ LVAD $=$ left ventricular assist device \\ $\mathrm{MCV}=$ monthly countervalue}

(Thoratec Corp, Pleasanton, CA) device to explore the advantages and the disadvantages in applying the paymentby-results approach to this type of innovative devices.

\section{MATERIALS AND METHODS \\ Design}

By using published information available on patients who received the HeartMate device, ${ }^{8}$ our analysis was aimed at determining the values of reimbursement associated with each patient according to the payment-by-results approach. ${ }^{4,5}$ The individual survival times (indSTs) after implantation for the published 68 patients receiving the HeartMate device ${ }^{8}$ were derived by a computerized analysis of the original Kaplan-Meier curve. ${ }^{9}$ In addition, the 11 survivors at the last time point of the published follow-up (45 months) were assigned an additional survival time (AST), thus resulting in an overall postimplantation survival of 45 months + AST.

The rationale behind our economic calculations was that the payment for each patient had to correspond to an amount of money equal to MCV for every month of survival lived by the patient beyond the final date of his or her life residual expectancy without an LVAD. Our base-case analysis was developed as follows: $\mathrm{MCV}=€ 5000$; no adjustment for quality of life (ie, utility $=1$ ); life expectancy without LVAD predicted at baseline, ie, before implantation, bLE $=150$ days or 5 months per patient [according to Park and colleagues ${ }^{8}$ )]; AST $=12$ months.

\section{Clinical Material and Survival Information}

The 68 patients treated with the HeartMate device (53 male, 97.1\% with New York Heart Association class IV, mean age of 66 years) have been described in detail in the original report. ${ }^{8}$ For these 68 patients, the indSTs reconstructed from the original Kaplan-Meier curve have been reported, ${ }^{9}$ along with information on how these data were extracted from the published survival curve. The value of AST $=12$ months, introduced in our base-case analysis, was determined by extrapolating the published survival curve to infinity according to the Gompertz method. ${ }^{10,11}$

\section{Basecase Analysis}

First, the data of each of the 68 patients were analyzed according to the following equation: individual reimbursement $=($ indST-bLE $) \times \mathrm{MCV}(\mathrm{Eq}-$ uation 1), where the values of indST and bLE are expressed in months and monetary parameters are expressed in Euros; individual reimbursement was set to 0 when bLE $>$ indST. Then, the average of these 68 reimbursements was calculated and its value was finally compared with the true price of the HeartMate device (currently $€ 75,000$ ).

\section{Sensitivity Analysis}

A first series of analyses was carried out by introducing utility as a quality-of-life adjustment for indSTs. The utility values were drawn from Moskowitz and colleagues, ${ }^{12}$ who reported a mean utility of 0.809 in LVAD recipients (with the \pm 1 standard deviation interval ranging from 0.945 to 0.673). In another sensitivity analysis, the value of bLE varied from 6 to 24 months, and in another analysis the value of AST assigned to the 11 survivors varied over the range from 2 to 36 months. In a further conclusive sensitivity analysis, benefits were discounted at $1.5 \%$ per year and costs were discounted at $3 \%$ per year. The authors had full access to the data and take responsibility for its integrity. All authors have read and agree to the article as written.

\section{RESULTS}

The mean reimbursement found in our basecase analysis of the 68 patients was $€ 82,426$ (range, $€ 0$ to $€ 250,000$ ). These 68 values were distributed as shown in Figure 1. The average value of $€ 82,426$ was close to the current price of the HeartMate device $(€ 75,000)$.

Sensitivity testing (Table 1) showed that the basecase "average" reimbursement of $€ 82,426$ was little influenced by variations in bLE; in contrast, the variations in utility had a more pronounced impact (Table 1).

The information on the 2 sensitivity analyses (assessing the effect of discounting or changes in AST, respectively) is not presented in this article because changes in these 2 variables did not introduce any meaningful change $(<15 \%)$ into our estimates of average reimbursement.

\section{DISCUSSION}

The most commonly used value-based methods for reimbursing innovative drugs or medical devices do not generally apply the payment-by-results approach but rely on the evidence that has accumulated about the "average" (or expected) incremental benefit of the innovative intervention. Because the growth of cost-effectiveness analysis has paralleled the growth of evidence-based medicine, cost-effectiveness has derived from evidence-based medicine a methodological attitude favoring "summary" estimates rather than the assessment of individual patients.

The traditional cost-effectiveness approach based on "average" effectiveness generally proceeds through 3 steps:

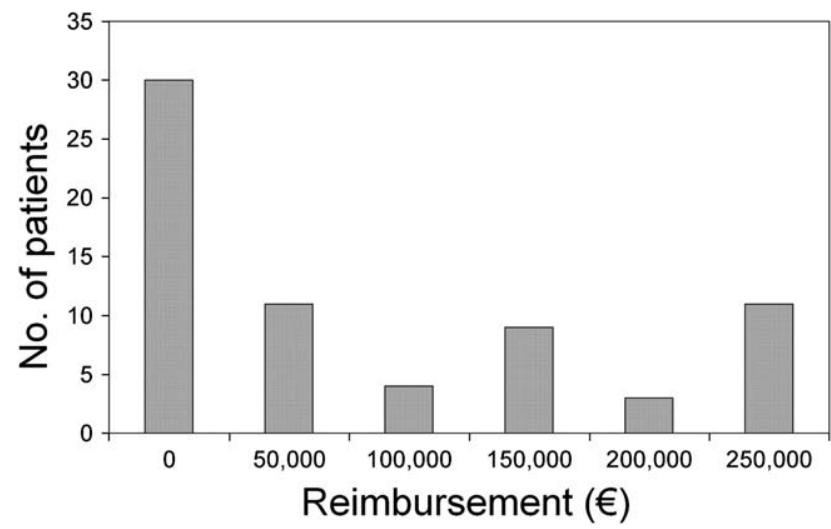

FIGURE 1. Distribution of the 68 values of reimbursement calculated according to the payment-by-results approach. The first bar on the left refers to reimbursements from $€ 0$ to $€ 24,999$, the second bar refers to reimbursements from $€ 25,000$ to $€ 74,999$, and so on. 
TABLE 1. Results of the sensitivity analyses in which utility and baseline life expectancy without left ventricular assist device were varied

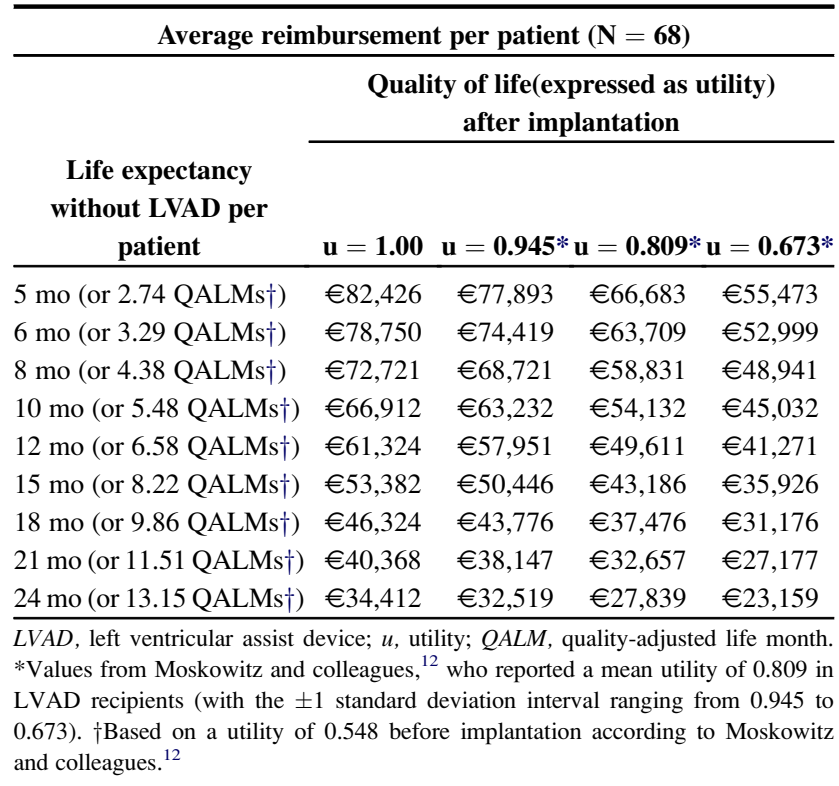

1. retrospective analysis of the "average" trial-based clinical effectiveness;

2. decision-making about reimbursements based on the "average" outcome; and

3. prospective application of the reimbursements defined in step (2) to the future cases of the "real world."

This approach works particularly well when the clinical evidence is sound mainly because, under these circumstances, both the national health system (or third payer) and the manufacturer are willing to share the same expectation about the treatment outcome.

In recent times, cases have emerged in which the national health system and the product manufacturer could not share the same expectation about the treatment outcome (eg, interferon for multiple sclerosis, ${ }^{13}$ bortezomib for multiple myeloma, ${ }^{14}$ and ranibizumab for macular degeneration ${ }^{15}$ ). Typically, the manufacturer had the tendency to fully trust the results of the (preliminary) clinical trials, whereas the national health system was more cautious and unwilling to fully accept the outcomes reported by the preliminary trials. At least in the United Kingdom, these cases have all been governed using the payment-by-results approach.

LVADs still have a premature body of effectiveness data, ${ }^{7}$ with the important exception of the HeartMate device. ${ }^{8}$ For this reason, in the present economic study the case of the HeartMate device was selected as a worked example to develop a payment-by-results model for the reimbursement of any type of LVADs and to test this model ex post against the large series of real cases already available for the HeartMate device.

The value-for-money principle states that the economic aim of health care systems is to purchase as much health as possible, and not to merely purchase drugs or devices. For example, in the area of oncology, it has recently been observed that "spending $\$ 2900$ per month in lapatinib (or $\sim \$ 100$ per day) does not purchase the $1250 \mathrm{mg}$ daily of the drug, but purchases the progression-free months gained by the patients as a result of the drug's efficacy.",16

Thus, a change in the mental attitude of health professionals seems to be needed, and this applies particularly to the area of medical devices. Accordingly, in the field of LVADs the belief that the money spent on this innovative treatment is intended to purchase the device should be discouraged because this money actually purchases the survival gain (resulting from the LVAD in the patient in whom the device is implanted).

In this framework, our model for the reimbursement of LVADs has been developed in full accordance to the valuefor-money principle and by giving preference to the payment-by-results approach because the therapeutic evidence for all LVADs except the HeartMate device is still limited.

Our proposed procedure has modeled the entire reimbursement procedure of LVADs on the basis of only 2 fundamental assumptions:

1. The value of $b L E$ (ie, the residual life expectancy without LVAD in the individual patient concerned): For the purposes of the present study, the best estimate that we found in the literature was 150 days (according to Park and colleagues ${ }^{8}$ ), and so this value was introduced in Equation 1 as a uniform parameter for all patients ("population method'). Interestingly enough, this point of our procedure can be further improved in prospective patient series by individualizing the value of bLE ("individualized method") according to the Seattle model; ${ }^{17,18}$ this individualization, however, requires the knowledge of approximately 20 staging parameters at baseline for each patient (that were unavailable for the 68 patients who received the HeartMate device in the context of our study).

2. The value of $M C V$ (ie, the societal economic countervalue of 1 month of life gained): Our analysis was run by setting $\mathrm{MCV}=€ 5000$, but of course our procedure can be run using other values of MCV. Higher values of MCV (eg, $€ 8000$ ) could be used as a means to encourage therapeutic innovation; this would imply the awareness by decisionmakers that the first $€ 5000$ for each quality-adjusted life month gained represents the standard value-based payment, whereas those exceeding the threshold of $€ 5000$ (eg, €3000) explicitly serve the purpose to support innovation. Experts in health economics have long agreed that each unit of survival gained should preferably be valued as a societal average without introducing any adjustment depending on the patient concerned. ${ }^{5,19-20}$

It is noteworthy that the above 2 parameters are fully independent of the particular type of LVAD implanted in the 
patient. Thus, the main application of our reimbursement model is not for the HeartMate device (for which the data of effectiveness are adequate), but for the other LVADs that are presently supported by less data regarding their effectiveness. ${ }^{7}$

It should also be noted that patients with end-stage heart failure unsuitable for transplantation generate follow-up costs that are not too dissimilar depending on whether or not they have received the LVAD. ${ }^{19}$ Thus, in economic terms the 2 main factors that generate the difference include the cost of the surgical implantation of the device $\left(\sim \$ 67,000,{ }^{19}\right.$ with year of costing $=2004$, which corresponds to $\sim € 50,000$ using the exchange rate of January 2004 reported by the www.oanda.org website) and the cost of the device itself. ${ }^{7}$

The payment-by-results approach can be implemented more easily in countries with a national public health care system than in countries where private health care prevails. In fact, most experiences in the area of payment by results have thus far taken place in the framework of national health care systems (eg, the reimbursement of bortezomib for multiple myeloma in the United Kingdom ${ }^{14}$ and of sorafenib for hepatocellular carcinoma in Italy; Messori A, personal communication, 2008). Despite that, the payment-by-results approach has recently attracted the interest of numerous health care experts in the United States who have contributed to the debate on this issue. ${ }^{4,19,21}$

On the one hand, the American health care system is characterized by a degree of heterogeneity that does not facilitate reimbursements based on value-for-money or payment-byresults and that favors instead the traditional fee-for-service approaches; in addition, the natural propensity of American insurance companies for administrative models of payment and not for evidence-based methods further complicates the application of this approach in the United States.

On the other hand, the increasing availability of innovative high-cost treatments in the health care marketplace emphasizes the need to devise methods that give more priority to innovative treatments with proven clinical benefit and less priority to those characterized by uncertain effectiveness. These methods, which cannot have a merely administrative basis, tend to force third payers (irrespective of their private or public nature) to face, on clinical and scientific grounds, the problem of differentiating between real clinical benefits and potential benefits and to recognize to innovation an economic countervalue proportional to the (incremental) clinical benefit.

As a result, a lively debate has been started in the United States to contrast the pros and the cons of the value-formoney approach ${ }^{19,21}$ and to examine the potential areas of application of the payment-by-results approach. ${ }^{4,21}$ One important advantage of LVADs is that, in assessing the effectiveness of these devices, survival is an undisputed end point for quantifying the benefit.

As new LAVDs other than the HeartMate device enter the market (eg, Jarvik [Jarvik Heart Inc, New York, NY], Novacor [World Heart Inc, Oakland CA], and Berlin Heart [Berlin
Heart AG, Berlin, Germany] devices), a difficult question will be posed to decision makers because a) not reimbursing them for lack or insufficiency of effectiveness data would negate these devices to patients and hamper the overall innovation process in this area; $b$ ) reimbursing them at the price claimed by the manufacturer would imply to make a decision on their price only on subjective grounds or by purely negotiable criteria, thus failing to apply the value-for-money principle. The application of a payment-by-results procedure (as described in the present report) therefore seems to be an appropriate solution to this problem.

Our article is not intended to discuss the local methods of payment that could be set up by individual institutions within the boundaries of the payment-by-results approach. Nevertheless, because this approach requires that health is purchased a posteriori (ie, after it has already been generated), the simplest solution is that the manufacturer of the LVAD is paid monthly for an amount of money equal to the MCV multiplied by the number of patients being treated after their bLE with the device during that month.

The average economic amount per device recognized by our analysis was, as expected, the result of a series of individual values distributed over a wide range (Figure 1). Variations over opposite extremes ranging from $€ 0$ to more than $€ 250,000$ pose an extraordinarily strong challenge to both the health care system and the manufacturer in which the outcome of the individual patient (along with his or her undisputable end point of survival) represents the key factor for the whole mechanism of payment.

The payment-by-results approach explicitly requires to change the way the hospital (or third payer) reimburses the vendor because, for example, the latter would be paid monthly at a rate of approximately 5000 euros per month for every month lived by the patient beyond his or her expected life expectancy without implant. These regular repetitive payments are already a standard in other areas of medicine, for example, home dialysis. ${ }^{22}$

It is true the first payment would be received by the vendor only when as many months have passed as is the patient's expected survival without implant (determined at baseline, ie, at the time of the implant). Regardless of the method for predicting the expected survival without implant (based on either population average $\operatorname{data}^{8}$ or an individualized model ${ }^{18}$ ), this lag in the initiation of the payments is in full accordance with the risk-sharing nature of this form of reimbursement. Of course, there would be no substantial distortion in the payment-by-results approach in the case of local deals or negotiations wherein a small proportion of the list price (eg, 10\%) is paid to the vendor at the time of the implant; this would prevent the extreme situation in which the vendor provides the device to the hospital but ultimately receives no payment at all for the device (ie, in cases of therapeutic failures with postimplant survival less than the patient's expected survival without implant). 
On the other hand, the vendor would be the stakeholder who could financially benefit more from a long-term success in the payment-by-results method. These cases are, however, also "favorable" for the hospital because the patient's improvement in survival (which is paid for) does not have an expected nature, but a real one.

In our proposal, life expectancy for patients without an implanted LVAD is handled either as a median "population" value drawn from the Kaplan-Meier curve ("population method'") or as an individualized covariate-adjusted estimate ("individualized method") determined by the application of the Seattle model (variables incorporated in the Seattle model ${ }^{18}$ include gender, age, New York Heart Association class, weight, ejection fraction, systolic blood pressure, medications, and clinical chemistry data).

Thus, our approach does not have any intermediate option between the first logical extreme where median survival is not adjusted for any patient's variable (ie, no adjustment for gender, no adjustment for age, and so on) and the other logical extreme where survival is adjusted for an array of patient-related variables (ie, the 8 variables of the Seattle model mentioned above).

Finally, it should be noted that survival data from HeartMate device implants include few female patients ${ }^{8}$ so that our "population method", could understate to some extent the baseline survival one might see today with current treatment modality in patients without an implanted LVAD with class IV heart failure.

Our sensitivity analysis was interesting particularly in regard to the effect of utility. A close scrutiny of the results shown in Table 1 supports the view that the average reimbursement associated with the utility of $0.809(€ 66,683)$ is actually representative of "real" outcomes so that one can even postulate that the average of $€ 66,683$ might paradoxically be "more basecase" than our basecase analysis.

One question arises on whether or not the economic countervalue of the benefit (determined according to Equation 1) should be intended to cover only the cost of the device (first case) or, alternatively, all the main sources of incremental cost (second case) inclusive of the cost of the device and the cost of surgery (approximately $€ 50,000^{23}$ ).

In the first case, if one makes reference to total costs and total reimbursements over a representative series of the number of patients receiving implants, the total cost for the number of devices at their current real price (total cost $=€ 75,000 \times \mathrm{N}$ ) is likely to be fully covered by the reimbursements determined according to our procedure (total reimbursement $=$ sum of $\mathrm{N}$ unpredictable individual reimbursements whose average is expected to be $€ 82,426$, ie, $€ 82,426 \times \mathrm{N}$ ).

In the second case, the total incremental cost for the number of patients receiving implants (total cost $=€ 75,000 \times \mathrm{N}$ $+€ 50,000 \times \mathrm{N}=€ 125,000 \times \mathrm{N}$ ) would not be fully covered by the reimbursements (total reimbursements $=$
$€ 82,426 \times \mathrm{N})$, and so a certain proportion of the total incremental cost (ie, $34 \%$ according to the above figures) would remain without coverage. Interestingly enough, the local health policy of our region of Tuscany currently seems to be in favor of the "first" case.

\section{CONCLUSIONS}

This report delineates an innovative procedure for the economic management of LVADs that seems to be without alternatives to ensure an appropriate allocation of economic resources, at least in this area of invasive cardiology. In Italy and many other countries, one factor that can increase the willingness of vendors to accept these innovative payment schemes is that LVADs are not presently included in any diagnosis-related group capable of covering their high acquisition cost. So, these innovative payment approaches can be the only way to open the market to these new devices and to allow patients to have access to this form of potential therapeutic advance.

\section{References}

1. Henry D, Hill S, Harris A. Drug prices and value for money. The Australian pharmaceutical benefits scheme. JAMA. 2005;294:2630-2.

2. Appleby J, Devlin N, Parkin D. NICE's cost effectiveness threshold. BMJ. 2007; 335:358-9.

3. Pearson SD, Rawlins MD. Quality, innovation, and value for money-NICE and the British National Health Service. JAMA. 2005;294:2618-22.

4. Garber AM, McClellan MB. Satisfaction guaranteed- " "payment-by-results" for biologic agents. N Engl J Med. 2007;357:1575-7.

5. Breckenridge A, Walley T. Risk sharing and payment by results. Clin Pharmacol Therap. 2008;83:666-7.

6. Messori A. Drug reimbursement policies: prospective vs. retrospective application of the value for money principle. eBMJ. 30 May 2008. Available at: http:// www.bmj.com/cgi/eletters/336/7658/1413. Accessed January 15, 2009.

7. Clegg AJ, Scott DA, Loveman E, Colquitt J, Hutchinson J, Royle P, Bryant J. The clinical and cost-effectiveness of left ventricular assist devices for end-stages heart failure: a systematic review and economic evaluation. Health Technol Assess. 2005;9:1-132.

8. Park SJ, Tector A, Piccioni W, Raines E, Gelijns A, Moskowitz A, et al. Left ventricular assist devices as destination therapy: a new look at survival. J Thorac Cardiovasc Surg. 2005;129:9-17.

9. Messori A. Methods for meta-analysis: reconstructing individual survival times through the analysis of Kaplan-Meier graphs. eBMJ. 15 Sep 2008. Available at: http://www.bmj.com/cgi/eletters/336/7658/1413\#201829. Accessed January 15, 2009.

10. Messori A, Becagli P, Trippoli S, Tendi E. A retrospective cost-effectiveness analysis of interferon as adjuvant therapy in high-risk resected cutaneous melanoma. Eur J Cancer. 1997;33:1373-9.

11. Mark DB, Hlatky MA, Califf RM, Naylor CD, Lee KL, Armstrong PW, et al. Cost effectiveness of thrombolytic therapy with tissue plasminogen activator as compared with streptokinase for acute myocardial infarction. N Engl J Med. 1995; 332:1418-24.

12. Moskowitz AJ, Weinberg AD, Oz MC, Williams DL. Quality of life with an implanted left ventricular assist device. Ann Thorac Surg. 1997;64:1764-9.

13. Napier JC, Francis R, Wright G. Shared scheme for assessing drugs for multiple sclerosis: cost effective provision of effective treatments for multiple sclerosis. BMJ. 2003;326:1212.

14. Jack A. Drug pricing—no cure, no cost. BMJ. 2007;335:122-3.

15. Dobson R. NICE introduces cost sharing scheme for blindness drug. BMJ. 2008; 337:538-9.

16. Trippoli S. Controversies in using lapatinib at reduced dosage with food. J Clin Oncol. 2007;25:5333. 
17. Mozaffarian D, Anker SD, Anand I, Linker DT, Sullivan MD, Cleland JG, et al. Prediction of mode of death in heart failure: the Seattle Heart Failure Model. Circulation. 2007; 116:392-8.

18. Levy WC, Mozaffarian D, Linker DT, Sutradhar SC, Anker SD, Cropp AB, et al. The Seattle Heart Failure Model: prediction of survival in heart failure. Circulation. 2006;113:1424-33.

19. Steinbrook R. Saying no isn't NICE-the travails of Britain's National Institute for Health and Clinical Excellence. N Engl J Med. 2008;359:1977-81.

20. Wu Y, Jin R, Gao G, Grunkemeier GL, Starr A. Cost-effectiveness of aortic valve replacement in the elderly: an introductory study. J Thorac Cardiovasc Surg. 2007;133:608-13.
21. Redberg RF, Walsh J. Pay now, benefits may follow-the case of cardiac computed tomographic angiography. N Engl J Med. 2008;359:2309-11.

22. Just PM, de Charro FT, Tschosik EA, Noe LL, Bhattacharyya SK, Riella MC. Reimbursement and economic factors influencing dialysis modality choice around the world. Nephrol Dial Transplant. 2008;23: 2365-73.

23. Clegg AJ, Scott DA, Loveman E, Colquitt J, Royle P, Bryant J. Clinical and costeffectiveness of left ventricular assist devices as destination therapy for people with end-stage heart failure: a systematic review and economic evaluation. Int $J$ Technol Assess Health Care. 2007;23:261-8. 